\title{
Riego y Usuarios del Agua en Chile. Desde la Revolución a la Evolución
}

\author{
José Luis Arumi ${ }^{1}$, Ovidio Melo ${ }^{1}$, Jorge Nuñez ${ }^{2}$ y Max Billib ${ }^{3}$ \\ ${ }^{1}$ Universidad de Concepción, Chile; 2Universidad de La Serena y Cazalac, Chile; ${ }^{3}$ University of Liebniz Hannover, \\ Alemania \\ jarumi@udec.cl,omelo@udec.cl,jnunez@cazalac.org, billib@iww.uni-hannover.de
}

\begin{abstract}
Resumen. El manejo del agua de riego en Chile se ha transformado siguiendo los procesos que han afectado a la sociedad chilena, pero manteniendo el principio de que el manejo del agua es controlado a través de las Organizaciones de Usuarios de Agua y que el Estado posee solamente un rol regulador. Durante un breve período de la historia de Chile se impusieron dos reformas, una destinada a modificar la tenencia de la tierra y otra que impuso un modelo neoliberal que trajo una legislación de aguas que separa estas de la tierra y trata su derecho como una propiedad privada. Las presiones producto de la globalización, las demandas de los mercados internacionales y las nuevas demandas de los actores sociales que requieren de una mejor calidad de vida y una mayor protección ambiental, llevan a la agricultura y el riego en Chile a estar en plena evolución hacia un sistema centrado en la optimización económica del recurso hídrico, y con el desafío de dar soluciones a las nuevas demandas por parte de la sociedad, relacionadas con la acceso a bebida y protección del medioambiente, entre otros usos.
\end{abstract}

Palabras Clave: Riego, Organizaciones de Usuarios de Agua, reforma agraria, Ley de agua, variabilidad climática

\begin{abstract}
The management of irrigation water in Chile has been transformed following the processes that have influence Chilean society, but always keeping the principle of which the management of the water is controlled by the Water Users' Organizations and that the State possesses only a regulatory role. During a brief period of the history of Chile there were imposed two reforms: one destined to modify the property of the land and the other one where a neoliberal model was impose who brought a water legislation that separates these from the land property and treats water rights as a private property. Pressures product of Globalization, demands of the international markets and the new demands of the social actors that are asking of a better quality of life and a major environmental protection, lead to the agriculture and the irrigation in Chile to being in full evolution towards a system focused on the optimization of the water resource, and with the challenge of giving solutions to the new demands on the part of the company, related to the drink and the environment, between other uses.
\end{abstract}

Keywords: Irrigation, Water User Organizations, Land reform, Water Law, Climate variability

\section{Introducción}

El manejo del agua de riego en Chile se ha transformado siguiendo los procesos que han afectado a la sociedad chilena, pero manteniendo el principio básico de que el manejo del agua es controlado a través de las organizaciones de usuarios de agua y que el estado posee solamente un rol regulador. Este principio se desarrolló a lo largo de la historia de Chile y nación por la influencia de la cultura Española introducida al país durante el período colonial. 
Durante un período muy breve de la historia de Chile se produjeron dos cambios que fueron impuestos por revoluciones ideológicas: la reforma agraria y la actual legislación de agua que posee Chile, ambos cambios han debido enfrentar la inercia social que significa la historia de Chile, pero además enfrentar nuevos desafíos productos de la globalización, la apertura de los mercados y las demandas sociales gatilladas por movimientos como los Indignados.

La suma de estas forzantes, así como el impacto de períodos de escasez causados por la variabilidad climática y la competencia por los recursos agua y suelo frente a otros sectores de la economía, han llevado a la agricultura chilena a enfrentar un proceso de evolución, que es irreversible.

El análisis que se presenta en este documento está basado en los resultados de la experiencia directa de algunos de los autores trabajando con los regantes, muchas horas de conversación e innumerables litros de café y cerveza.

\section{Características de los recursos hídricos en Chile}

A lo largo de su territorio Chile posee una particular distribución de disponibilidad de recursos hídricos, pues esta va desde el desierto más árido del planeta en el norte, a zonas en la Patagonia Austral donde precipita 335 días en el año. Para comprender las características del territorio Chileno, es necesario considerar la tectónica de placas pues Chile se encuentra ubicado en el borde occidental del continente sudamericano donde se produce la subducción de la Placa de Nazca bajo la placa Sudamericana. Este proceso de subducción, produjo el levantamiento de la Cordillera de la Costa hace 400 millones de años (Figura 1a). Posteriormente, hace 70 millones de años se produjo el levantamiento de la Cordillera de los Andes que forzó el desarrollo de una red de drenaje hacia el Océano Pacífico; esta red de drenaje cruzó la antigua Cordillera de la Costa a través de fallas geológicas produciendo cañones que fueron después cubiertos de sedimentos para formar los típicos valles de los ríos que cruzan dicha cordillera (Figura 1b). Posteriormente, producto de grandes fracturamientos se hundieron grandes bloques que al ser rellenados formaron lo que actualmente conocemos como la Pampa del Tamarugal y el Valle Central de Chile (figuras 1c y 1d).

Como resultado de la subducción, la Cordillera de los Andes se formó a lo largo de la costa Occidental de Sudamérica y actúa como una gran barrera orográfica que intercepta la circulación general de los vientos (Figura 2). El movimiento de los sistemas frontales que se desplazan de oeste a este en la zona austral, desviándolos hacia el norte y generando los sistemas de tormentas frontales que producen la lluvia que alimenta la zona central de Chile. Por otro lado los sistemas frontales provenientes del Atlántico en el Norte de Sudamérica son interceptados por el Altiplano Andino, que impide que la lluvia descargue en la zona de Atacama. Es así que el efecto de la Cordillera de Los Andes sobre la circulación general de los vientos, produce la existencia de grandes zonas áridas en el Sur del Perú, Norte de Chile y Sur de Argentina, en lo que es conocido como Diagonal de Aridez de Sudamérica. 
El efecto combinado de la distribución de humedad y de la formación del territorio produce las cuatro grandes macrozonas de Chile: El Norte Grande, que es híper árido; el Norte Chico, que es árido; la Zona Central que va desde Santiago, que es semi-árido y llega a Puerto Montt, que ya es una región húmeda; y finalmente la zona Austral donde abundan las precipitaciones. Esto es relevante para este artículo, porque la agricultura no se desarrolló masivamente en la zona híper árida (con la salvedad de algunos oasis y valles como el de Lluta), se desarrolló en forma limitada en la zona árida y no requiere riego en las zonas húmedas. Por esta razón, la cual la agricultura de riego en Chile se desarrolla principalmente entre La Serena (Latitud $30^{\circ} \mathrm{S}$ ) y Los Ángeles (Latitud $37^{\circ} \mathrm{S}$ ).

Como Chile posee un clima Mediterráneo que se caracteriza por que las lluvias se concentran en invierno ( $85 \%$ del total precipitado anual ocurre entre el 15 de abril y el 15 de septiembre); la producción de muchos productos como las frutas, hortalizas y cultivos industriales necesita del riego para lograr obtener una productividad adecuada.
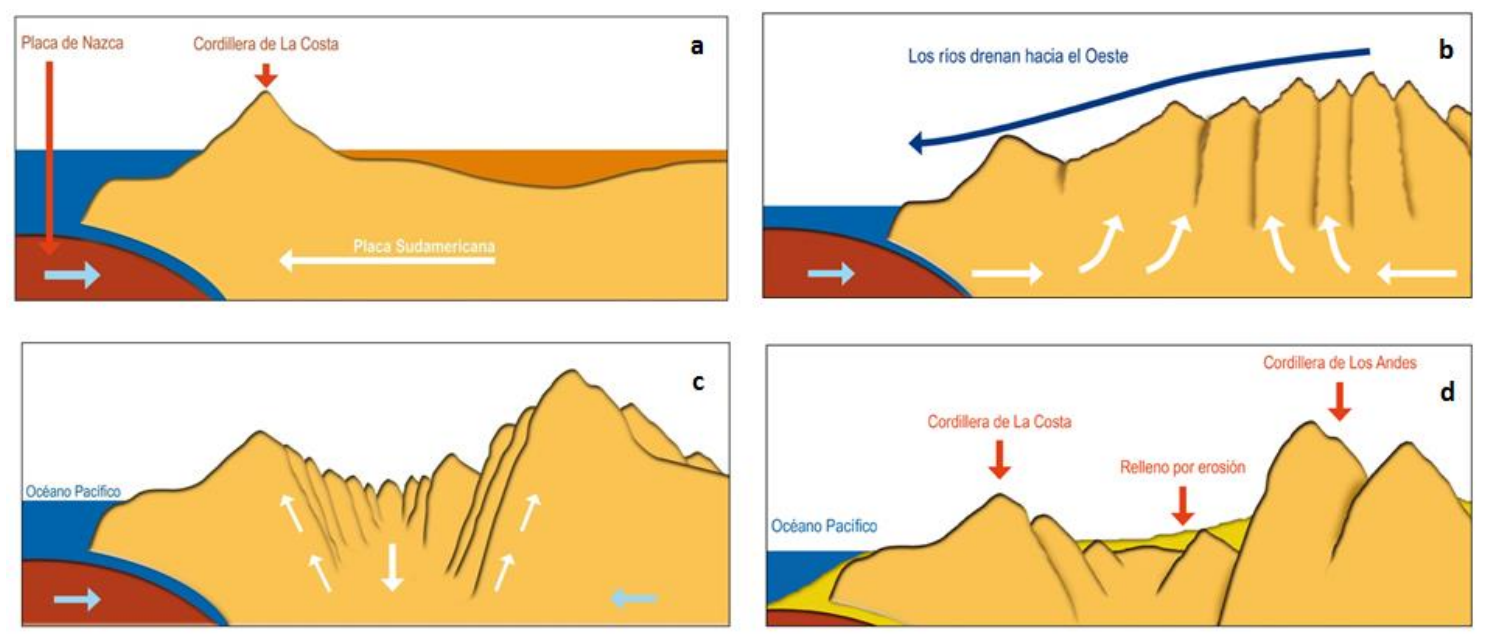

Fig. 1. Proceso de formación del territorio de Chile.

Sin embargo, debido a que las cuencas chilenas son pequeñas estas no puede almacenar grandes reservas de agua y por ello los ríos poseen un caudal muy variable. Por ejemplo, si bien el río BíoBio tiene un caudal promedio de unos $1000 \mathrm{~m} 3 / \mathrm{s}$, en invierno su caudal puede superar los $10.000 \mathrm{~m} 3 / \mathrm{s}$ debido a los grandes frentes de mal tiempo y en verano, cuando no llueve, se acerca a los $100 \mathrm{~m} 3 / \mathrm{s}$.

Estas diferencias en las magnitudes de los caudales a lo largo del año se ven aún más complicadas con las enormes diferencias que existen entre los diferentes años húmedos y secos. Chile se ve muy afectado por forzantes climáticos como las Oscilación del Sur del Niño (ENSO) y la Oscilación Decadal del Pacífico (PDO) que han generado períodos de abundancia y escasez de agua que sólo se ha entendido recientemente (Rubio-Alvarez and McPhee, 2010; Núñez 2014).

Todo lo anterior produce una gran presión en la agricultura de riego, pues no sólo deben lidiar con el manejo del agua durante los años secos, sino que también deben proteger la infraestructura durante los años excepcionalmente húmedos cuando se producen grandes tormentas que generan inundaciones catastróficas. 


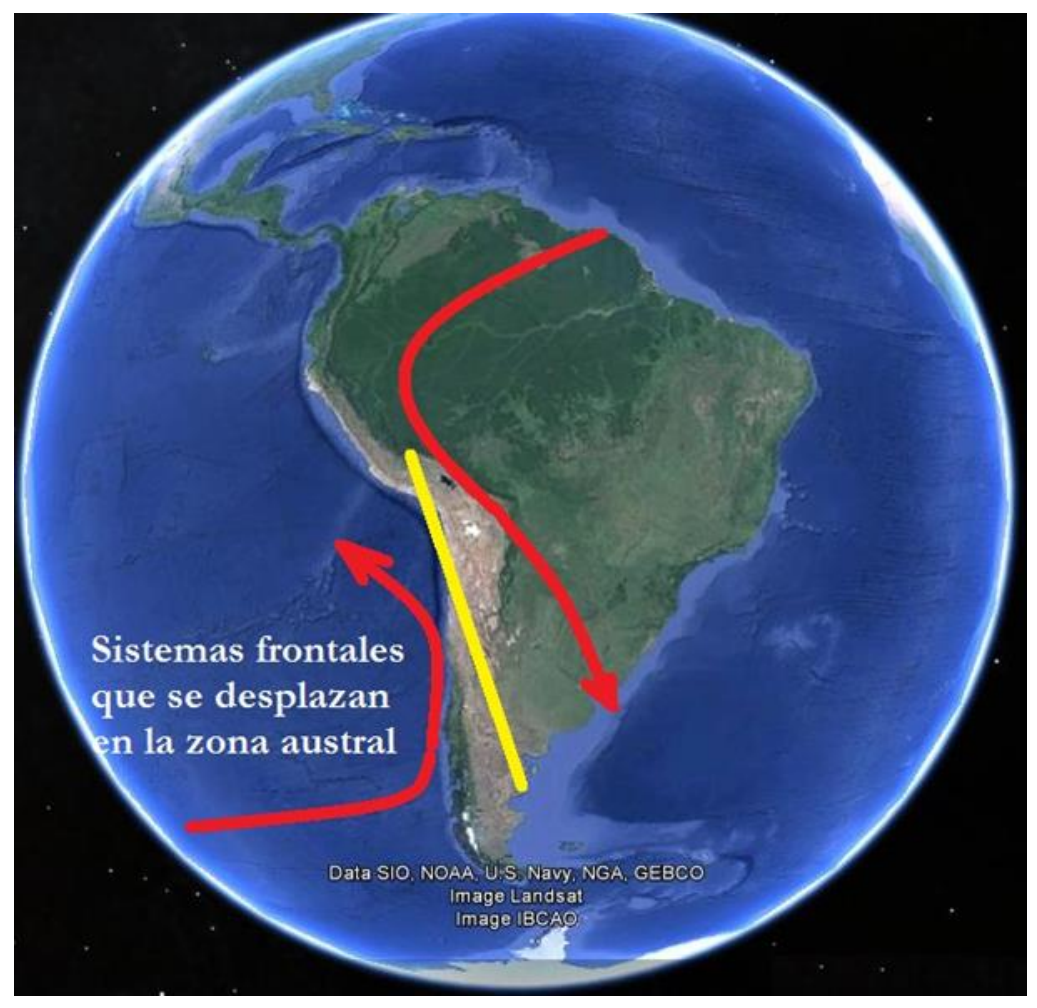

Fig. 2. Esquema del fecto de la Cordillera de los Andes sobre la circulación general de los vientos en Sudamérica. La línea amarilla representa la diagonal de aridez de Sudamérica

(adaptado de Garreaud, 2009, usando de base una imagen de Google Earth)

\section{Antecedentes sobre el manejo del agua de riego en Chile}

Como se mencionó anteriormente la agricultura de riego se desarrolla en Chile principalmente entre las ciudades de la Serena y Los Ángeles. En este sentido es importante destacar que existe una interesante actividad agrícola asociada al riego en los valles y Oasis del Norte de Chile, pero debido a que este territorio fue anexado a Chile después de la Guerra del Pacífico (1884) y además a que esta agricultura está desarrollada por población originaria, su modelo es diferente al que se maneja en la zona central de Chile y no será incluido dentro del análisis de este documento, principalmente porque escapa de la experiencia de los autores.

El modelo de gestión de las agua de Chile, se desarrolló principalmente para el manejo del agua de riego y en menor medida para el abastecimiento de agua de la población. En este sentido este modelo, desarrollado en la zona central de Chile, no considera elementos provenientes de las culturas heredadas de los pueblos originarios, pues estas fueron desplazadas por la introducción de las prácticas españolas en Chile durante el período colonial. En este período, se otorgaban mercedes de agua destinadas a diferentes usos, principalmente el agrícola y el abastecimiento de las ciudades. Estas mercedes de agua eran entregadas por el Gobernador (representante del Virrey del Perú) y por el Presidente de la Real Audiencia. 
En relación a la administración, el Gobernador y el Cabildo designaban Jueces de Agua, quienes distribuían el recurso en sectores de escasez. El Cabildo, además, se encargaba de la ejecución de las obras necesarias para abastecer de agua a las ciudades. Muchas disposiciones legales que afectaban a las mercedes y a la distribución de las aguas se encontraban contenidas en las Leyes de Indias, y otras de aplicabilidad más directa, en disposiciones y dictámenes de los Cabildos (Retamal et al. 2012).

Con el advenimiento de la independencia de Chile, llegaron cambios vinculados con la administración pública, lo que conllevó modificaciones relativas a la gestión del agua, pero que desde un punto de vista sustancial y práctico, no implican grandes cambios. Primero surge la figura del Presidente de la República, quien tiene la facultad de otorgar las mercedes de agua. Además, los Intendentes y Gobernadores también cuentan con dicha atribución. Por su parte, los Intendentes nombraban a los Jueces de Agua, cuya función era la distribución del recurso, al igual que en el período anterior, (Retamal et al., 2012).

Según Donoso et al., (2004), el primer texto patrio que regula el uso de las aguas data de 1819, y pertenece al Presidente Bernardo O'Higgins, quien promulga un Decreto Supremo donde define la dimensiones de un regador, formas de venta y responsabilidad de las bocatomas.

Más adelante, en 1855 se promulga el primer Código Civil, que contaba con normativas relacionadas con el agua, que no diferían sustancialmente con lo descrito anteriormente. Luego, se promulgaron diversas ordenanzas municipales destinadas principalmente a: (1) solucionar conflictos de distribución de mercedes de agua en las cuencas de la zona norte y central de Chile; y (2) ordenar el uso de las mercedes de agua mediante la creación de Juntas de Vigilancia. En consecuencia, los jueces de Agua poseían la autoridad para solucionar los conflictos, y las Juntas de Vigilancia acordaban entre sus miembros la distribución de las aguas según las mercedes otorgadas por el Estado. Es importante destacar que las Juntas de Vigilancia de esa época constituyen los primeros intentos para organizar a los usuarios de aguas entorno a la gestión de la demanda del agua, modelo que perdura hasta estos días (Retamal et al., 2012).

En 1908 se promulga la ley de asociaciones de canalistas, que surge debido al impulso económico de comienzos del siglo XX, que aumentó la demanda de agua y con ello la presión al Estado de tomar un rol más activo. Se crea la "Inspección General de Regadío" dependiente de la Dirección de Obras Públicas que tuvo como primera misión la construcción de grandes obras de riego.

Este modelo de gestión de aguas consideraba como eje de la administración del recurso a las asociaciones de canalistas, entidades conformadas por los "dueños del agua", lo que constituye un avance significativo en cuanto al modelo actual (Melo, 2014). El Código de 1951 promueve la creación de estas organizaciones que pasan a ser supervigilaras por el Departamento de Riego. Adicionalmente, se reforzó la resolución de conflictos ente usuarios al interior de las OUA como primera instancia y en caso de no resolución se recurría a los Tribunales de Justicia. En esencia este procedimiento es el que actualmente opera en el sistema actual de gestión del agua en Chile (Retamal et al., 2012). 


\section{Imposición de dos revoluciones}

Entre los 18 años que abarcan desde 1967 a 1985 se modificó sustancialmente el modelo de manejo de agua que se había desarrollado en Chile desde la fundación de Santiago (1541). Efectivamente a partir de la aparición del Código de aguas de 1967, se introdujeron dos grandes revoluciones: Primero la reforma Agraria, que buscó distribuir las propiedad de las tierras y la imposición de un modelo Neoliberal por la Dictadura de Pinochet.

La aparición del Código de Aguas de 1967 aumentó de manera considerable la participación del Estado en la administración de los recursos hídricos y que tenía como propósito apoyar los objetivos de la reforma agraria. Este cuerpo legal cambió sustancialmente la naturaleza jurídica del derecho de aprovechamiento de agua, toda vez que le da un carácter de derecho real administrativo, donde el Estado concede el uso del bien nacional de uso público sujeto a normas del derecho público. En consecuencia, concede el uso de las aguas pero no su dominio sobre ellas. En ese sentido, los derechos de agua pasan a ser administrativos y caducables. La re-asignación se somete a la planificación del Estado mediante la aplicación de la "tasa de uso racional y beneficioso", suprimiéndose el listado de usos preferentes y dejando como prioritarios la bebida y el agua potable (Donoso, 2004).

Sin embargo, con la promulgación del Código de Aguas de 1981 (o incluso antes, con el golpe de Estado de 1973), se modifica sustancialmente la gestión de las aguas hacia un modelo neoliberal, reduciendo al mínimo la función de planificación que tenía el Estado.

El Código de Aguas de 1981 eliminó toda posibilidad de caducar por parte de la administración los derechos de agua, suprimiéndose las normas que pudiera debilitar la propiedad sobre los mismos. Ejemplo de esto último era la posibilidad de declarar área de racionalización y suspender los derechos existentes, o restringir los usos más o menos libremente durante los períodos de sequía (artículos 35 y 332 del Código de Aguas de 1967) (WGP, 2004).

Este modelo se deriva de la Constitución Política de 1980, que en su artículo 19, N²4 asegura a todas las personas el derecho de propiedad en sus diversas especies sobre toda clase de bienes corporales e incorporales. Expresa en su inciso final que "Los derechos de los particulares sobre las aguas, reconocidos o constituidos en conformidad a la ley, otorgan a sus titulares la propiedad sobre ellos". Este modelo es único en su especie, ya que se reconoce al agua como una mercancía totalmente transable (Bauer 2004, 2005, 2009, Hearne y Donoso, 2005, Larraín, 2010, citados por Retamal et al., 2012), y porque los DAA son entregados a particulares gratuitamente, a perpetuidad, sin necesidad de justificar a qué se destinará su uso y pueden ser transferidos como cualquier otro inmueble.

\subsection{La reforma agraria}

Producto de los procesos sociales dominantes a nivel internacional, en Chile se generó la reforma agraria entre los años 1962 y 1973 que fue un proceso destinado a entregar tierras a los campesinos, expropiándoselas a los grandes terratenientes y generando una nueva clase de agricultor que son los denominados "parceleros", quienes forman la actual pequeña agricultura campesina de Chile. El nivel de intensidad de expropiaciones y las características del proceso fue bien diferente a lo largo del país y según Enríquez (1987) casi el 40 \% de los beneficiados vendió sus tierras y volvieron a ser obreros agrícolas. Este proceso de reforma fue detenido bruscamente por el Golpe Militar de 1973. 
El tema de la reforma agraria ha sido y es un tema que despierta discusión en Chile, por ello no es intención de este trabajo abordar todos sus aspectos sino que analizar el impacto que tuvo en la estructura de riego de Chile.

Por un lado, existe un consenso entre los agrónomos y otros profesionales ligados a la agricultura que el proceso de la reforma agraria gatilló un cambio profundo en la agricultura nacional, pues al perder las tierras los grandes agricultores fueron motivados a cambiar su patrón productivo desde la agricultura extensiva (ganadería o trigo) a una agricultura intensiva y más productiva. Sin embargo también existe un consenso en que producto del ambiente político de la época la agricultura campesina fue dejada de lado, generando un proceso de empobrecimiento en dicho sector.

Por otro lado, en su experiencia profesional, los autores han podido constatar un efecto poco documentado de la reforma agraria sobre la estructura de riego en la zona central de Chile.

La mayor parte de la red de canales existente en la zona Central de Chile se construyó por iniciativa de los grandes hacendados en la primera parte del Siglo XX. Grandes obras civiles que involucraron la construcción de túneles, puentes y grandes volúmenes de movimiento de tierra fueron hechas a un gran costo económico y también humano. Es así como al inicio de la década de 1960 se contaba con una importante infraestructura que permitía el riego de casi un millón de hectáreas.

Un grave error del proceso de reforma agraria, fue desvincular a los parceleros de la infraestructura de riego, ellos sentían que "el canal sigue perteneciendo al patrón y que era responsabilidad de él mantenerlo". Por otro lado, para el patrón, o el gran hacendado, el canal ya no era su problema, sino que era problema de los "Huasos" que a los que les habían entregado sus tierras.

Esta situación dejó a la infraestructura de riego en un limbo que duró casi 20 años y que sólo se enfrentaría al inicio de la década de 1990, en el gobierno del Presidente Aylwin, al retorno de la democracia en Chile.

\subsection{La Ley de Riego}

En el año 1985, se promulgó la Ley 18.450 de Fomento a la Inversión Privada en Obras de Riego y Drenaje cuya creación impulsó la transformación de la agricultura Chilena desde un patrón extensivo a un patrón intensivo. Esta Ley se transformó en un importante incentivo a la incorporación de tecnologías que permitieron sustentar nuevos productos agrícolas. De esta forma, el patrón del Riego cambió, desplazando los métodos tradicionales existentes en 1960 por sistemas tecnificados, que actualmente ocupan más del 50\% de la superficie de riego actual en Chile, regando nuevos productos más rentables, pero más sensibles a la disponibilidad de agua.

Un cambio importante a la aplicación de esta Ley lo realizó el Gobierno de Patricio Aylwin, quien modificó la interpretación original de la Ley, orientada originalmente a empresarios, para incluir la posibilidad de subsidiar fuertemente la incorporación de tecnologías en la agricultura campesina. De esta forma, a partir de 1990 se inició un fuerte desarrollo en la incorporación de sistemas tecnificados y en la recuperación de la infraestructura de riego, que había sufrido de años de abandono. Es así como en los siguientes 20 años se hizo un 
gran esfuerzo en la recuperación y expansión de la infraestructura de riego, así como en la implementación de sistemas presurizados.

Cifras oficiales, obtenidas a partir de los Censos Agropecuarios de 1997 y 2007 reflejaron un incremento de cobertura de los sistemas de riego presurizados de un $9 \%$ a un $30 \%$, lo que demuestra el éxito de la implementación de esta Ley.

Desde el punto de vista hidrológico, la incorporación de sistemas de alta eficiencia permitió al agricultor expandir su superficie de riego plantando laderas con frutales, pues él es el propietario de sus derechos de agua y por lo tanto si ahorra agua puede aumentar su superficie de riego. Esta condición causa una disminución delos caudales de retorno producido por la baja eficiencia de los métodos tradicionales de riego. Esos caudales de retorno ya no están disponibles en la parte baja de la cuenca para los agricultores que usaban esa agua.

En ese sentido, la revolución que el Código de Aguas de 1981 vino a consolidar y acrecentar, se debió fundamentalmente a la concesión del "derecho de propiedad" y no al mercado como los esperaban los ideólogos del Código. Esto ha sido demostrado empíricamente. De hecho, los ideólogos de esa legislación fundamentaban que las bondades del mercado de aguas iban a determinar que los ahorros producidos por el aumento de la eficiencia de riego producida por la incorporación de tecnologías de riego iban a ser vendidos al mercado. Este es el mejor ejemplo de la mala interpretación de los creadores de esa legislación a la Tragedia de los Comunes en torno al agua.

Nuevos tiempos implican nuevos problemas y la Ley de Riego ha sido usada para enfrentarlos mediante la modificación de su reglamento. Es así que con el Gobierno de Ricardo Lagos se incluyó la posibilidad de subsidiar el uso de tecnologías para enfrentar los emergentes problemas de calidad de agua y con el Gobierno de Sebastián Piñera se incluyeron iniciativas para fomentar la incorporación de telemetría, automatización y energías renovables.

Es así como un instrumento legal, desarrollado inicialmente bajo un modelo neoliberal y destinado a las grandes Empresas agrícolas, probó ser un instrumento flexible que ha podido ser usado por los distintos gobiernos como una herramienta para enfrentar distintos problemas que han buscado solucionar en torno al riego.

\section{La evolución}

Durante los últimos 20 años han ocurrido una serie de cambios, que atrapados en lo cotidiano no percibimos, pero al mirar atrás nos causan asombro. La reducción de los costos de viaje y transporte, la irrupción de la internet y de los medios de comunicación han generado una era de globalización que nos ha afectado en todos los aspectos de nuestras vidas y por supuesto la Agricultura y el riego en Chile no escaparon de ella.

Con la firma de innumerables tratados internacionales, Chile abrió grandes oportunidades pero también se sometió a distintos marcos regulatorios. Por ejemplo las exigencias del Eurogap marcaron las necesidades de invertir en solucionar los problemas de calidad de agua que no habían sido un tema en muchos años. 
Otro tema que irrumpió fuertemente ha sido la variabilidad climática que marcó significativamente la producción agrícola en Chile a partir del año 2007 cuando se registró una serie de 7 años de escasez hídrica. Sólo recientemente hemos podido identificar que Chile es fuertemente impactado por mecanismos climáticos como son la Oscilación del Sur del Niño y la Oscilación Decadal del Pacífico (Rubio-Álvarez y McPhee, 2010; Núñez, 2014). De hecho estos forzantes climáticos han generado períodos de abundancia y escasez de agua, como los descritos en la Biblia con la historia del Faraón que soñaba con siete vacas gordas que eran devoradas por siete vacas flacas. Desafortunadamente, no contamos con la visión de construir los graneros para almacenar alimentos en la época de las vacas flacas. De hecho, no existe memoria colectiva de la sequía similar que existió en la década de 1930 y peor aún, nuestra actual Legislación de Aguas se instauró en la década de 1980, que fue un período de abundancia de agua y que fue cuando se repartieron la mayor parte de los derechos de agua.

Un aspecto importante que hay que destacar es que al promulgarse la Ley de riego en 1985, fue necesario realizar cálculos hidrológicos para estimar "Seguridades de riego" que corresponde a caudales asociados a una probabilidad de excedencia del $85 \%$, eso quiere decir que los proyectos se evalúan económicamente considerando el caudal mínimo que ocurre durante el 85\% del tiempo. Un problema que se ha suscitado con el reciente período de escasez hídrica, es que todos estos cálculos se realizaron sin considerar la variabilidad climática, usando el supuesto de que la disponibilidad de agua siempre sería similar a la que existía en la década de 1980, que fue el período más húmedo de los últimos 60 años.

Ante esto, la pregunta clave es: quién asume la responsabilidad del mal cálculo del riesgo de falla (caudal 85\%). En un sector económico exportador, debiesen haber sido los agricultores (Figueroa, 1999), fundamentalmente el empresario agrícola el que debió haber calculado e integrado ese riesgo (como el financiero o cualquier otro) dentro de su análisis de costos. Sin embargo, fue la Ley 18.450 quien, de alguna manera, se entrometió definiendo el procedimiento de cálculo de ese riesgo (Q 85\%). He ahí el meollo del asunto. Ya que el sector agrícola exportador usufructuario de la Ley de Riego podría ahora pasarle la factura al Estado por no actualizar su procedimiento de cálculo del riesgo hidrológico.

Lo anterior demuestra que es necesario fortalecer el conocimiento en materia de recursos hídricos. Esto implica, por ejemplo, mejorar la red hidrométrica nacional, de modo de facilitar la gestión a nivel institucional y particular. Además, es fundamental la implementación de tecnologías más avanzadas en el manejo de la información.

Se debe considerar la integración de diferentes sectores con una mirada interdisciplinaria, de modo de fomentar la investigación en áreas relacionadas con el cambio climático, la disponibilidad del recurso, la integración de: (1) las diferentes fases del ciclo hidrológico (glaciares, ríos, acuíferos); (2) la oferta y demanda del recurso; (3) los diferentes usos; (4) de la calidad y cantidad; y (5) la percepción de los diferentes actores relacionados.

Debido a las características particulares tan demandantes del agua, la coordinación intra- e intersectorial es muy importante dentro de este sector. El agua es móvil; su abastecimiento es incierto y variable, y puede provenir de diferentes fuentes; y hay una interdependencia predominante entre sus usuarios. 


\section{Comentarios finales}

El sistema de gestión de aguas chileno, no es consecuencia sólo de la promulgación del Código de Aguas de 1981, sino más bien el resultado de un largo proceso que se inicia en tiempos de la colonia, caracterizado en tres ejes fundamentales: (1) sistema concesional en que el Estado otorga un derecho de aprovechamiento a los particulares que lo soliciten, dándole el estatus de propiedad privada sobre el derecho otorgado; (2) la concepción de un Estado subsidiario y con escaza participación en cuanto a la gestión del recurso; (3) la existencia de organizaciones de usuarios del agua conformadas sólo por titulares de derechos de aprovechamiento, sin ningún tipo de participación de los usuarios sin derechos, de las futuras generaciones o de la sociedad en su conjunto.

Este largo camino hacia la "neo-liberalización" del modelo, fue quitándole atribuciones al Estado en cuanto al derecho de agua, y por el contrario aumentando la seguridad jurídica a los particulares y con ello el estatus de propiedad privada, la que se encuentra protegida constitucionalmente. En este sentido, la actual legislación consagra una total libertad para el uso del agua a que se tiene derecho, pudiendo los particulares destinarla al uso que deseen, sin mayor participación del Estado, dejando en manos del mercado y de las organizaciones de usuarios del agua (OUAs) las principales funciones relativas a la gestión de las aguas.

Actualmente la agricultura debe enfrentar un cambio en la tendencia de disponibilidad de agua, pues ahora se encuentran enfrentado un período de escasez. Además deben competir con usos del agua y del suelo que son más rentables que a producción agrícola (por ejemplo el desarrollo inmobiliario, la minería y la generación de energía). Por ello, dadas las condiciones actuales que existen en Chile, la agricultura debe considerar nuevas especies que sean competitivas en los mercados internacionales, por lo que cada vez es más común encontrar plantaciones frutales y sistemas de riego industrializados que utilizan aguas subterráneas.

El manejo del agua de riego en Chile se ha transformado siguiendo los procesos que han afectado a la sociedad chilena, pero manteniendo el principio de que el manejo del agua es controlado a través de las Organizaciones de Usuarios de Agua y que el Estado posee solamente un rol regulador.

En consecuencia, el riego en Chile está en plena evolución hacia un sistema centrado en la optimización económica del recurso hídrico, y con el desafío de dar soluciones a las nuevas demandas por parte de la sociedad, relacionadas con la bebida y el medioambiente, entre otros usos.

Agradecimientos. Los autores expresan su agradecimiento a Conicyt por el financiamiento dado a través de los proyectos Fondecyt-BMBF-PCCI1-2031 y Conicyt/Fondap/15130015.Además es importante mencionar nuestro agradecimiento a la National Sciences Foundation de Estados Unidos por el soporte dado a través del proyecto Acequia Water Systems Linking Culture and Nature: Integrated Analysis of Community Resilience to Climate and Land-Use Changes. NSF-Proposal 1010516. 


\section{Referencias}

Donoso, G.; A., Jouravlev, H. Peña, E., Zegarra (2004): Mercado (de derechos) de agua: experiencias y propuestas en América del Sur. CEPAL. División de Recursos Naturales e Infraestructura. Santiago de Chile.

Enriquez, M.E. (1987): "Reforma agraria en Chile", Revista de Geografía Norte Grande, 14: 6165.

Figueroa, J.S. (1999): "Sequía extrema y responsabilidad del empresariado. Revista de Derecho Administrativo Económico. 1 (2): 383-385

Global Water Partnership (2004): Investigación sistémica sobre regímenes de gestión del agua. el caso de Chile. http://www.eclac.cl/DRNI/proyectos/samtac/InCh01304.pdf

Núñez J., Rivera D., Oyarzún R., Arumí J.L., (2014): Influence of Pacific Ocean multidecadal variability in the distributional properties of hydrological variables in north-central Chile. Journal of Hydrology

Retamal, M.R., Melo, O., Arumi, J.L., Parra O. (2013): "Sustainable water governance and climate change in Chile: from the sectorial management system to an integrated one?" en Rivera D. (Ed.) Chile: Environmental, Political and Social Issues, pp 33-68, Nova Science Publishers, Inc. 2012.

Rubio-Álvarez, E. y J. McPhee (2010): "Patterns of spatial and temporal variability in streamflow records in south central Chile in the period 1952-2003", Water Resources Research, 46, W05514, doi:10.1029/2009WR007982. 